\title{
Desafios de enfermeiras frente à saúde do homem na atenção básica
}

\author{
Challenges of nurses in front of man's health in basic care \\ Desafíos de las enfermeras frente a la salud del hombre en cuidados básicos
}

Recebido: 11/01/2021 | Revisado: 13/01/2021 | Aceito: 15/01/2021 | Publicado: 19/01/2021

\author{
Claudia Moreira de Lima \\ ORCID: https://orcid.org/0000-0001-9864-7651 \\ Universidade do Estado de Mato Grosso, Brasil \\ E-mail: cml_claudiamoreira@hotmail.com \\ Hérica Aparecida Albuquerque de Arruda \\ ORCID: https://orcid.org/0000-0002-4977-4766 \\ Universidade do Estado de Mato Grosso, Brasil \\ E-mail: herica_dtno@hotmail.com \\ Roseany Patrícia Silva Rocha \\ ORCID: https://orcid.org/0000-0002-2295-5321 \\ Universidade do Estado de Mato Grosso, Brasil \\ E-mail: roseanyrocha1@gmail.com \\ Ronaldo Antonio da Silva \\ ORCID: https://orcid.org/0000-0002-1962-3182 \\ Universidade do Estado de Mato Grosso, Brasil \\ E-mail: ronaldoantonioenf@gmail.com \\ Daniela Luzia Zagoto Agulhó \\ ORCID: https://orcid.org/0000-0002-3434-9863 \\ Universidade Federal de Mato Grosso, Brasil \\ E-mail: dani.zagoto@outlook.com \\ Dayane dos Santos Souza Magalhães \\ ORCID: https://orcid.org/0000-0001-9999-3396 \\ Hospital Universitário Júlio Muller/UFMT/EBSERH, Brasil \\ E-mail: dayane.souza.enf@gmail.com
}

\begin{abstract}
Resumo
É sabido que há uma reduzida frequência de usuários do sexo masculino nos serviços da Atenção Básica, muitas são as pressuposições, para que essa situação aconteça. Objetivo: esta pesquisa teve como objetivo descrever os desafios enfrentados por enfermeiras atuantes na atenção básica em ralação à saúde do homem. Método: Tratou-se de um estudo qualitativo, realizado com quatro enfermeiras atuantes nas Estratégias de Saúde da Família, aos quais se aplicou um roteiro de entrevista semiestruturada, em junho de 2019. Para análise dos dados, optou-se pelo emprego da análise de conteúdo da temática das narrativas. Resultados: evidenciou-se que fatores sociais, culturais e institucionais, e a não implantação da política estão entre os motivos que contribuem para que os homens não busquem assistência nos serviços da atenção básica. Conclusão: Assim, conclui-se que é necessário o cuidado à saúde do homem seja fomentado nesse contexto e que os profissionais enfermeiros atuantes na atenção básica considerem as suas singularidades, visando compreender a política e adotar estratégias que visem a sua implementação efetiva.
\end{abstract}

Palavras-chave: Saúde do homem; Saúde; Enfermagem; Atenção primária à saúde.

\begin{abstract}
It is known that there is a reduced frequency of male users in the Primary Care services, there are many assumptions for this to happen. Objective: this research aimed to describe the challenges faced by nurses working in primary care in relation to men's health. Method: It was a qualitative study, carried out with four nurses working in the Family Health Strategies, to whom a semi-structured interview script was applied, in June 2019. For data analysis, we opted for the use of the analysis of content of the narrative theme. Results: it was evidenced that social, cultural and institutional factors, and the non-implementation of the policy are among the reasons that contribute for men not to seek assistance in primary care services. Conclusion: Thus, it is concluded that it is necessary to promote men's health care in this context and that professional nurses working in primary care consider their uniqueness, in order to understand the policy and adopt strategies aimed at its effective implementation.
\end{abstract}

Keywords: Men's health; Gender and health; Nursing; Primary health care.

\section{Resumen}

Se sabe que existe una menor frecuencia de usuarios masculinos en los servicios de Atención Primaria, hay muchos supuestos para que esto suceda. Objetivo: esta investigación tuvo como objetivo describir los desafíos que enfrentan las enfermeras que trabajan en atención primaria en relación con la salud del hombre. Método: Fue un estudio 
cualitativo, realizado con cuatro enfermeras que laboran en las Estrategias de Salud de la Familia, a quienes se les aplicó un guión de entrevista semiestructurada, en junio de 2019. Para el análisis de datos se optó por el uso del análisis de contenido del tema narrativo. Resultados: se evidenció que los factores sociales, culturales e institucionales, y la no implementación de la política se encuentran entre las razones que contribuyen a que los hombres no busquen asistencia en los servicios de atención primaria. Conclusión: Así, se concluye que es necesario promover la atención de la salud masculina en este contexto y que los profesionales de enfermería que laboran en la atención primaria consideren su singularidad, a fin de comprender la política y adoptar estrategias encaminadas a su efectiva implementación.

Palabras clave: Salud de los hombres; Salud; Enfermería; Primeros auxilios.

\section{Introdução}

A forma que o homem se relaciona com aspectos relacionados aos cuidados em saúde tem sido apresentada de maneira negativa pela literatura científica, sendo a falta de procura por atendimento e a falta de preocupação com a própria saúde reflexo do aumento nos índices de morbimortalidade (Chaves et al., 2018). No cotidiano, os homens procuram o serviço de saúde quando a doença já está instalada, o que gera aumento nos gastos em saúde devido à complexidade do quadro, representando assim, uma problemática para a saúde pública do país (Brasil, 2008).

Porém, pensar em ações de cuidado como essencialmente femininas, ou excluí-las da realidade masculina, é fazer generalizações, assim transpondo o interesse da sociedade brasileira, o Ministério da Saúde (MS), aprovou em agosto de 2008 a Política Nacional de Atenção Integral à Saúde do Homem (PNAISH). Essa política representa importante avanço no que tange a saúde do homem no país e reconhece os fatores socioculturais que determinam a vulnerabilidade desses às doenças, compreendendo a realidade singular masculina nos diversos contextos, tanto político-econômica quanto nos socioculturais (Martins; Malamut, 2013; Teixeira et. al., 2014; Oliveira et. al., 2015).

Dentre os s objetivos da política, destacam os que têm como finalidade oferecer e facilitar o acesso a um conjunto de ações de promoção, prevenção, assistência e recuperação da saúde, alicerçado na atenção básica, em que o enfoque é a promoção de saúde e a prevenção de agravos, aumentando a expectativa de vida desse público e reduzindo os índices de morbimortalidade, devido as causas preveníeis dessa população (Martins \& Malamut, 2013; Teixeira et. al., 2014; Oliveira et. al., 2015).

No contexto de cuidado na atenção básica, o enfermeiro atua como gestor da Estratégia de Saúde da Família (ESF), sendo este profissional o elemento da equipe de saúde que mais tempo interage com o cliente, tendo entre suas funções o papel fundamental na promoção da saúde e na prevenção das doenças e outros agravos à saúde, além de privilegiar a educação em saúde, influenciar a aquisição de hábitos saudáveis, e investigar novas motivações e de outros fatores determinantes do comportamento (Alvarenga et al., 2012).

Tendo em vista que a equipe de enfermagem se preocupa e se empenha cada vez mais em melhorar a assistência humanizada e de qualidade, levantou-se como perguntas de pesquisa: Quais os desafios enfrentados pela enfermeira atuante na atenção básica em relação a saúde do homem? e Quais as ações estratégicas são elaboradas e desenvolvidas visando incentivar a adesão masculina neste ambiente de saúde? Sendo assim, este estudo teve como objetivo descrever os desafios enfrentados por enfermeiras atuantes na atenção básica em ralação à saúde do homem.

\section{Metodologia}

Trata-se de um estudo do tipo descritivo, com abordagem qualitativa. $\mathrm{O}$ estudo descritivo está relacionado à descrição das características, propriedades ou relações existentes na comunidade, grupo ou realidade pesquisada. Para pesquisas de caráter amplo, estes estudos tornam-se adequados, visto que permitem tarefas de elaboração clara do problema e da hipótese 
como tentativa de solução. E a abordagem qualitativa compreende uma junção de diversas técnicas de interpretação que pretendem descrever e decodificar os componentes de um sistema complexo de significados (Cervo, Bervian \& Silva, 2007).

O estudo teve como participantes quatro enfermeiras atuantes nas Estratégias de Saúde da Família localizadas em um município da região médio norte do estado de Mato Grosso, Brasil, que possui sete Estratégias de Saúde da Família (ESF) na área urbana e três na área rural que são denominadas de acordo com o nome do bairro onde se localizam, compondo-se: ESF Central, ESF Pedregal, ESF Bairro da Ponte, ESF, Buriti, ESF Bom Jesus, ESF Pé Branco, ESF Jardim Alvorada, ESF Bojuí, ESF Caeté e ESF Deciolândia.

A pesquisa foi realizada nas ESFs localizadas na área urbana do município de Diamantino, por se tratar de uma questão de conveniência de localidade e locomoção para realizar a pesquisa, fazendo parte da pesquisa então as ESF's: ESF Central, ESF Pedregal, ESF Bairro da Ponte, ESF, Buriti, ESF Bom Jesus, ESF Pé Branco e ESF Jardim Alvorada.

Para seleção foram considerados como critérios de inclusão: Ser graduado em enfermagem; Possuir Experiência profissional na atenção básica maior ou igual a 1 ano; estar ativo em sua função na ESF; e aceitar participar da pesquisa assinando o Termo de Consentimento Livre Esclarecido (TCLE).

Os sujeitos foram considerados em número suficiente para gerar a reincidência das informações, com inclusões sucessivas e satisfatórias de profissionais para uma discussão densa das questões formuladas, ou seja, a quantidade numérica de entrevistados utilizada no estudo não interferiu na qualidade dos resultados obtidos, pois a pesquisa qualitativa enfoca as informações e o aprofundamento na análise dos dados. Assim, a amostra não buscou uma representatividade numérica, mas sim um aprofundamento da temática.

O trabalho de campo ocorreu em junho de 2019 com auxílio de um roteiro norteador elaborado pelos autores com perguntas abertas para entrevista semiestruturada. As entrevistas individuais com auxílio do roteiro permitiram maior interação entre pesquisador e depoente, permitindo assim maior riqueza de informação possível a respeito do tema pesquisado.

As narrativas obtidas nas entrevistas foram gravadas e transcritas na integra, e submetidas à análise de conteúdo do tipo temática de Minayo (2014), seguindo os seguintes passos: pré-análise, que compreende a escolha dos materiais a serem analisados; exploração do material, onde foram identificados os núcleos textuais; e por fim, tratamentos e interpretação dos dados que foram analisados e discutidos de acordo com a literatura referente à temática em apreço. Nesse sentido, a partir da análise dos dados empíricos emergiram quatro categorias temáticas, a saber: Fragilidades no atendimento à saúde do homem; Momentos pontuais para busca por saúde pelo homem; Homens como sujeitos de cuidado e os estereótipos de gênero. Fragilidades no atendimento à saúde do homem; e Incompatibilidade de horários com a atividade laboral.

Para o desenvolvimento deste estudo foram respeitados os preceitos éticos e legais de pesquisas que envolvem seres humanos preconizados na Resolução $n^{\circ}$. 466/2012 do Conselho Nacional de Saúde (Brasil, 2008), assim como a resolução 311/2007 do Conselho Federal de Enfermagem. Assim, o estudo foi apreciado e aprovado pelo Comitê de Ética em Pesquisa da Universidade de Cuiabá (CEP/UNIC), sob protocolo 3.420.216 de 2019. A fim de garantir o anonimato dos sujeitos participantes, os nomes próprios foram substituídos por nomes de flores.

\section{Resultados e Discussão (pode ser separado ou junto)}

Em relação ao perfil dos participantes do estudo, as quatro enfermeiras eram do sexo feminino, com idade que compreende entre 37 a 45 anos, com tempo de graduação em enfermagem entre 12 a 20 anos, com especialização voltada predominantemente para a área de atenção básica, atuando na gerência e assistência neste nível de atenção. Apresentam experiência profissional de atuação na ESF, variando de 1 a 15 anos, sendo todos de vínculo estatutário e uma carga horaria ocupacional de 40 horas semanais. 
No que tange as ações desenvolvidas nas unidades de forma geral, estas englobam atividades de grupo de Hipertensão Arterial Sistêmica e Diabetes Mellitus, sendo realizado também atividades em saúde de forma individual com ações voltadas a saúde da criança e saúde da mulher. No que diz respeito especificamente em relação as ações voltadas para a saúde do homem, predominam o caráter de demanda livre ou em atividades desenvolvidas durante o mês de novembro, alusivo à saúde do homem.

Corroborando com nosso achado, um estudo apontou que na dinâmica de atendimento preconizada pelos programas de saúde do MS, a atenção a saúde do homem é comtemplada nos programas como hiperdia e demanda livre. Além disso, outro estudo realizado na zona norte da cidade do Rio de Janeiro, também identificou falha no atendimento à saúde do homem na atenção primaria, pois nos serviços investigados não eram desenvolvidos programas ou atividades, direcionadas especificamente para este público, fator esse que influência nas dificuldades de interação das necessidades de saúde dos homens, com a organização das ações de saúde das unidades de atenção primária (Silva et al., 2012).

A análise das unidades temáticas oriundas das narrativas das enfermeiras participantes da pesquisa revelou um campo amplo de dificuldades para a inserção do homem nas ações da atenção básica de saúde, apresentados nas categorias: Fragilidades no atendimento à saúde do homem; Momentos pontuais para busca por saúde pelo homem; Homens como sujeitos de cuidado e os estereótipos de gênero; e Incompatibilidade de horários com a atividade laboral.

\subsection{Fragilidades no atendimento à saúde do homem}

Esta categoria temática apresenta e discute as dificuldades para implementação da PNAISH enfrentadas na vivência das enfermeiras participantes do estudo. As narrativas revelam que a prática de educação em saúde no âmbito da saúde do homem é restrita, ao relatarem que não há capacitação/instrumentalização voltados para esse fim, dificultando, assim, as ações de saúde para atender às necessidades dessa clientela, o que pode ser tido como um impeditivo para alcançar um cuidado de excelência à saúde deste público. As narrativas apresentadas a seguir exemplificam essa análise:

\section{Rosa - "não, nunca foi ofertado[...] não somos treinados para isso" [...]. \\ Margarida - "não, nesse periodo que trabalho na atenção básica não houve capacitação". \\ Violeta - "não, nunca participei de uma capacitação". \\ Girassol - "não, nunca participei".}

Nesse sentido, destaca-se que a falta de "capacitação contínua" apontada pelas participantes relaciona-se a não existência de infraestrutura e organização de trabalho acerca das especificidades da saúde masculina. Desse modo, sabe-se que esta é de responsabilidade da organização laboral como também do trabalhador do sistema de saúde, principalmente se considerarmos que o perfil epidemiológico da população muda e que há doenças emergentes e reemergentes. Nesta perspectiva, aponta-se o déficit no processo de abordagem e de cuidar/cuidado da população masculina por parte dos profissionais, além da ausência de acolhimento ou acolhimento pouco atrativo está relacionado à frágil qualificação profissional para lidar com o segmento masculino (Oliveira et al., 2015).

Ressalta-se, portanto, que um dos objetivos da PNAISH é a promoção de saúde, informação/comunicação e a qualificação dos profissionais de saúde, assegurando o incentivo a capacitação dos profissionais de saúde atuantes na atenção básica (Brasil, 2008). Para Julião e Weigelt (2011), faltam meios adequados para o desenvolvimento de estratégias voltadas para a saúde do homem nas unidades básicas de saúde, entre eles, capacitação de pessoal, material didático sobre o assunto, espaço físico, condições de acesso para realizar exames, dentre outros. 
Assim, sabe-se que a aquisição de novos conhecimentos é estimulada a partir da estratégia de educação continuada realizada com estes profissionais. E pode representar uma iniciativa essencial para ampliar o cuidado direto ao público masculino, pois auxiliara o profissional a tornar-se mais ativo no que se expõe a saúde do homem, aliando a produção de conhecimento com uma visão humanitária (Bezerra et al., 2012). Além disso, a identificação das barreiras institucionais é um importante ponto para a proposição estratégica de medidas que venham a promover o acesso dos homens aos serviços da atenção primária, a fim de resguardar a promoção e a prevenção como eixos fundamentais de intervenção neste nível de atenção à saúde (Brasil, 2008).

\subsection{Momentos pontuais para busca por saúde pelo homem}

Quanto aos aspectos relacionados à problemática do estudo voltados a estratégias de inserção da clientela masculina na atenção básica, a análise dos conteúdos temáticas oriundos dos relatos das enfermeiras participantes da pesquisa, revelou um campo restrito de estratégias voltado a este público, onde as estratégias de inserção dos homens perpassam por três aspectos que estão vinculados a doença, aos encontros de grupos e a campanhas desenvolvidas.

Segundo Schraiber e colaboradores (2010), a inclusão dos homens em ações de saúde é um desafio para os profissionais da atenção básica, já que esse público não reconhece a importância do cuidado e da preservação do seu corpo no âmbito da saúde como algo inerente a sua própria condição biológica. E as práticas de saúde estão sendo realizadas também no sentido de não considerar a totalidade com um olhar holístico por parte do profissional, pois, na prática cotidiana de trabalho são realizados atendimentos parciais a esta parcela populacional, onde muitas delas estão voltadas a algumas estratégias de saúde especificas, conforme apresentado pelas narrativas a seguir:

Rosa - “[...], procuram quando a patologia já está instalada mesmo, ou no hiperdia” [...].

Violeta - “[...], homens são público menor, agora quando são desenvolvidas as campanhas, [...], reuniões de hiperdia sempre são a maioria" [...], durante a semana a procura pelo atendimento é para vacinas".

De acordo com Julião e Weigetel (2011), para que haja uma adesão dos homens com idade entre 20 e 59 anos, é necessário que os mesmos busquem mais os serviços de saúde, não somente nos períodos de campanha ou reuniões de grupo. Destaca-se a adoção de práticas que estimulem a participação masculina nas ações de promoção da saúde e prevenção de agravos, com planejamento e implementação de abordagens que possibilitem a valorização e a construção do sujeito como ser crítico frente à realidade, assim, garantindo o empoderamento deste no âmbito da saúde (Couto et. al., 2010). Logo, a estratégia de considerar e inserir a população masculina como sujeitos que precisam de cuidados no contexto da atenção básica, representa um desafio para a mudança no processo de trabalho em saúde para usuários do gênero masculino, mas tem o potencial de ampliar as práticas de cuidado envolvidas nas ações realizadas pelos profissionais da equipe de enfermagem.

\subsection{Homens como sujeitos de cuidado e os estereótipos de gênero}

O machismo se constrói, se exprime e se mantém, no decorrer da história da humanidade nas estruturas sociais, indo além da vontade individual de cada homem. É notória que a educação dada ao homem é diferente daquela dada a mulher. Pois enquanto a mulher é cuidada e educada com a ideia de que é, e sempre será um ser que precisa ser protegido o tempo todo, ao homem é sempre pregada a ideia de protetor e invulnerável (Fonseca, 2018). Nesse estudo, as narrativas das enfermeiras apontam que no que tange a relação do homem com a própria saúde e com os serviços que prestam cuidados em saúde, perpassa por sentimentos e expressões incorporadas a partir do machismo: 
Rosa - "o homem se julga muito machão[...] ele nunca fica doente" [...].

Margarida- "o homem, se sente muito o todo poderoso, menos vulnerável, [...] doença nunca vai atingi ló".

Girassol- "[...] o homem tem a questão de machismo, pelo fato de ser o mantenedor da casa, [...] medo de perde o emprego [...] acredito que homem tem essa dificuldade de autocuidado pela questão do machismo mesmo" [...].

Sabe-se que a vulnerabilidade da saúde do homem tem influências culturalistas aos "pré-conceitos" existentes em suas mentes de que são seres inabaláveis, indestrutíveis e que não podem adoecer, fazendo com que dessa forma não procurem a unidade de saúde. Isso faz com que quando busquem por atendimento, situação que ocorre majoritariamente quando a dor se torna insuportável, ou ainda quando a doença já está instalada (Schraiber et. al., 2010; Pereira; Nery, 2014; Oliveira et. al., 2015).

Estudo que corrobora com esses achados, buscou comparar a busca por atendimento de saúde entre o público feminino e masculino e os resultados aprontaram um percentual de 40,3\% para as mulheres e 28,4\% para os homens, tendo como justificativa para a procura pela mulher a prevenção, enquanto que para os homens por causa de algum agravo a saúde (Pinheiro et al., 2002). Em complementar a esse achado, estudos identificaram que a presença dos homens foi semelhante ou até superior quando comparadas a presença da mulher neste ambiente apenas em atendimentos de odontologia, vacinação, curativos e nas farmácias (Couto et. al., 2010; Albuquerque et. al., 2014; Pereira et. al., 2014; Trilico et. al., 2015).

Este fato pode ser justificado pelo motivo que os homens buscam por tratamento alternativo quando percebem alguma anormalidade, e nas farmácias, por exemplo, os homens conseguem expor mais facilmente os seus problemas, sendo também está uma maneira de tratamento rápido, que muitas vezes satisfaz o objetivo almejado, que é o alívio da dor. Além disso, no que se refere ao cuidado, este é reconhecido socialmente como inerente ao sexo feminino, desde a infância inclusive, e em contrapartida, os meninos desde a infância são moldados a serem fortes. Assim temos de um lado, o ser feminino como alguém que cuida da saúde, e do outro lado o masculino que não (re)conhece a necessidade de cuidados em relação a saúde (Couto et. al., 2010; Albuquerque et. al., 2014; Pereira et. al, 2014).

\subsection{Incompatibilidade de horários com a atividade laboral}

Ainda na perspectiva da identificação das dificuldades apontadas pelas enfermeiras com vistas a inserção dos homens em ações desenvolvidas na atenção básica, as narrativas apontam para o horário de funcionamento da unidade de saúde com um aspecto importante vinculado a oferta de serviços a este público, uma vez que o atual formato de funcionamento dificulta a ida ou até permanência do homem neste nível de atenção, conforme os relatos:

Rosa- "o município não oferta um horário diferenciado, devido ao horário laboral a população masculina não procura a unidade".

Margarida - "[...] na minha opinião a grande maioria desse público possui déficit de autocuidado, [...], mas a grande maioria queixa que por trabalharem não podem buscar esse atendimento [..]".

Conforme evidenciado nas narrativas o horário de funcionamento da atenção básica, por coincidir com o horário das atividades laborais da clientela masculina, torna-se um empecilho na busca pelo serviço por esse público (Moreira; Fontes; Barboza, 2014). As atividades laborativas são reconhecidas como prioridade para este grupo populacional, fazendo com que a prevenção e promoção em saúde ocupe segundo plano (Trilico et. al., 2015). Nesse sentido, Oliveira e colaboradores (2015), reafirmam que a coincidência de horários de atendimento do serviço de saúde com a disponibilidade de tempo do homem que 
trabalha dificulta o acesso e uso dos desses serviços, especialmente da atenção básica, fator este apontado por em outros estudos (Schraiber et. al., 2010; Moreira et. al., 2014).

Nesta perspectiva há poucas unidades de saúde que oferecem atendimento ampliado em formato de 24 horas, aos finais de semana ou terceiro turno (noite), o que impossibilita a ida de pessoas que trabalham no mercado formal, pois não podem se ausentar com a frequência exigida uma vez que isso poderá implicar na estabilidade do emprego. Nesse estudo, uma das enfermeiras apontou que essa oferta diferenciada de atendimento poderia influenciar de forma positiva no cuidado voltado ao público masculino, como expressa a narrativa a seguir:

\section{- "acredito que a oferta do terceiro turno, aumentaria a quantidade de atendimento aos homens”.}

A narrativa destaca o conhecimento da profissional enfermeira em relação a essa possibilidade de ofertar o cuidado em outro turno. Desse modo, esse profissional, assim como os demais, precisa atuar de forma ativa em conjunto com os gestores de saúde na avaliação da demanda e dos benefícios que essa incorporação poderá trazer para a saúde dessa população (Moreira; Fontes; Barboza, 2014).

\section{Considerações Finais}

O estudo permitiu evidenciar que se faz relevante analisar e reconfigurar a organização do trabalho e o processo laboral do sistema de saúde, pois embora haja a PNAISH que fundamenta o cuidado ao homem, há necessidade de capacitação dos profissionais de saúde, sobretudo dos enfermeiros, para que esses possam assistir à população masculina, atendendo a suas demandas a partir de suas particularidades.

Além disso, constatou-se que é preciso desconstruir a ideia de invulnerabilidade no homem, e esse pode ser um fator que impede a procura por atendimentos de prevenção nos serviços de saúde, de forma que esses se sintam à vontade e mais acolhidos. Considera-se a necessidade do desenvolvimento de novos estudos como este a fim de subsidiar estratégias que respondam aos anseios e às necessidades do público masculino em todas as suas fases de vida.

Neste contexto este estudo é de relevância, a se considerar o quanto a saúde pública no Brasil precisa evoluir, principalmente quando os indivíduos em questão são os homens, moldados pela cultura que os levam a exercer condutas perigosas a sua saúde, e podem provocar consequências que poderiam ser evitadas. Especificamente diante da problemática que envolve dificuldades e estratégias de inserção dos homens na atenção básica, com vista a desvelar a dinâmica dos serviços ofertados e das ações realizadas.

Por fim, considera-se que não há pretensão de se esgotar o assunto nesta pesquisa, e sim servir de auxílio a futuras pesquisas que acendem ao tema, e auxiliando aos demais, espera-se que o estudo possa contribuir para pensar estratégias de inserção desta clientela na atenção primária.

\section{Referências}

Albuquerque, G. A., Leite, M. F., Belém, J. M., Nunes, J. F. C., Oliveira, M. A., \& Adami, F. (2014). O homem na atenção básica: percepções de enfermeiros sobre as implicações do gênero na saúde. Escola Anna Nery, 18(4), 607-14. http://dx.doi.org/10.5935/1414-8145.20140086.

Alvarenga, W. A., Silva, S. S., Silva, M. E. D. C., Barbosa, L. D. C. S., \& Rocha, S. S. (2012). Política de Saúde do Homem: perspectivas de enfermeiras para sua implementação. Revista Brasileira de Enfermagem, 65(6), 929-35. https://www.scielo.br/pdf/reben/v65n6/a07v65n6.pdf.

Beck, C. L. C., Lisbôa, R. L., Tavares, J. P., Silva, R. M., \& Prestes, F. C. (2009). Humanização da assistência de enfermagem: percepção de enfermeiros nos serviços de saúde de um município. Revista Gaúcha de Enfermagem, 30(1), 54-61. https://seer.ufrgs.br/RevistaGauchadeEnfermagem/article/view/5102/65.

Bezerra, A. L. Q., Queiroz, E. S., Weber, J., \& Munar, D. B. (2012). O processo de educação continuada na visão de enfermeiros de um hospital universitário. Revista de Enfermagem, 14(3), 618-25. https://revistas.ufg.br/fen/article/view/12771/13431. 
Research, Society and Development, v. 10, n. 1, e38810111885, 2021

(CC BY 4.0) | ISSN 2525-3409 | DOI: http://dx.doi.org/10.33448/rsd-v10i1.11885

Cervo A. L., Bervian, P. A., \& Silva R. Metodologia Científica. Edições 6; 2007.

Brasil. (2008). Ministério da Saúde. Departamento de Ações Programáticas Estratégicas. Política Nacional de Atenção Integral à Saúde do Homem: princípios e diretrizes. http://www.saude.gov.br/sas/portarias.

Chaves, J. B., Silva, M. L. V., Bezerra, D. S., \& Fernandes, S. C. S. (2018). Saúde Masculina: O Paralelo Prevenção X Cuidado á Luz da Teoria da Ação Planejada (TAP). Saúde e Pesquisa,11(2), 315-324. http://dx.doi.org/10.17765/1983-1870.2018v11n2p315-324.

Couto, M. T., Pinheiro, T. F., Valença, O., Machin, R., Silva, G. S. N., Gomes, R., Schraiber, L. B., \& Figueiredo, W. S. (2010). O homem na atenção primaria à saúde: discutindo invisibilidade a partir da perspectiva de gênero. Interface (Botucatu),14(33), 257-270. https://doi.org/10.1590/S141432832010000200003 .

Fonseca, L. (2018). Homens e cuidado: uma outra família? Pontifícia Universidade Católica de São Paulo;

Gonçalves, A. et. al. (2010). Saúde do Homem: um novo conceito de Saúde Pública. Santos.

Julião, G. G., \& Weigelt, L. D. (2011). Atenção à Saúde do Homem em uma Unidade Estratégia de saúde as famílias. Revista de Enfermagem da UFSM, 1(2), 144-152. https://periodicos.ufsm.br/reufsm/article/view/2400/1743.

Martins, A. M., \& Malamut, B. S. (2013). Análise do discurso da política nacional de atenção integral à saúde do homem. Saúde e Sociedade, 22(2), 429-440. https://doi.org/10.1590/S0104-12902013000200014.

Minayo, M. C. S. (2014). O desafio do conhecimento: pesquisa qualitativa em saúde. (14a ed.), Hucitec.

Moreira, R. L. S. F., Fontes, W. D., \& Barboza, T. M. (2014). Dificuldades de inserção do homem na atenção básica a saúde: a fala dos enfermeiros. Escola Anna Nery, 18(4), 615-621. https://doi.org/10.5935/1414-8145.20140087.

Oliveira, M. M., Daher, D. V., Silva, J. L. L., \& Andrade, S. S. C. A. (2015). A saúde do homem em questão: busca por atendimento na atenção básica de saúde. Ciências em saúde coletiva, 20(1), 273-278. https://doi.org/10.1590/1413-81232014201.21732013.

Pereira, L. P., \& Nery, A. A. (2014). Planejamento, gestão e ações à saúde do homem na estratégia de saúde da família. Escola Anna Nery Revista de Enfermagem, 18(4), 635-643. https://doi.org/10.5935/1414-8145.20140090.

Schraiber, L. B., Figueiredo, W. S., Gomes, R., Couto, M. T., Pinheiro, T. F., Machin, R., Silva, G. S. N., \& Valença, O. (2010). Necessidades de saúde e masculinidades: atenção primária no cuidado aos homens. Caderno de saúde pública, 26(5), 961-70. https://doi.org/10.1590/S0102-311X2010000500018.

Silva, L. D., Beck, C. L. C., Dissen, C. M., Tavares, J. P., Budó, M. L. D., \& Silva, H. S. (2012). O enfermeiro e a educação em saúde: Um estudo bibliográfico. Revista Enfermagem da UFSM, 2(2), 412-419. https://doi.org/10.5902/217976922676.

Teixeira, D. C., Brambilla, D. K., Adamy, E. K., \& Krauzer, I. M. (2014). Concepções de enfermeiros sobre a política nacional de atenção integral à saúde do homem. Trabalho educação e saúde, 12(3), 563-576. https://doi.org/10.1590/1981-7746-sip00009.

Trilico, M. L. C., Oliveira, G. R., Kijimura, M. Y., \& Pirolo, S. M. (2015). Discursos masculinos sobre prevenção e promoção da saúde do homem. Trabalho educação saúde, 13(2), 381-95. https://doi.org/10.1590/1981-7746-sip00015. 